\title{
Criminologie
}

\section{Les homicides entre délinquants : une analyse des conflits qui provoquent des règlements de comptes}

\section{Gilbert Cordeau}

Volume 22, numéro 2, 1989

Le milieu criminel

URI : https://id.erudit.org/iderudit/017280ar

DOI : https://doi.org/10.7202/017280ar

Aller au sommaire du numéro

Éditeur(s)

Les Presses de l'Université de Montréal

ISSN

0316-0041 (imprimé)

1492-1367 (numérique)

Découvrir la revue

Citer cet article

Cordeau, G. (1989). Les homicides entre délinquants : une analyse des conflits qui provoquent des règlements de comptes. Criminologie, 22(2), 13-34.

https://doi.org/10.7202/017280ar
Résumé de l'article

Certain conflicts between offenders over their illicit activities end in the murder of one of the protagonists. These homicides are refered to as settling of accounts. This article examines why and under what circumstances offenders choose to solve their differences by a settling of accounts. Three categories of conflicts between offenders were identified. The 444 cases of settling of accounts ending with 531 victims that occured in Quebec from 1970 to 1986 were analyzed within those categories. The findings indicate that these homicides occured in cases where the stakes were very high. This study also shows why certain criminal activities and certain illicit markets give rise to more settling of accounts than others. 


\title{
LES HOMICIDES ENTRE DÉLINQUANTS : UNE ANALYSE DES CONFLITS QUI PROVOQUENT DES RĖGLEMENTS DE COMPTES 1
}

Gilbert Cordeau ${ }^{2}$

\begin{abstract}
Certain conflicts between offenders over their illicit activities end in the murder of one of the protagonists. These homicides are refered to as settling of accounts. This article examines why and under what circumstances offenders choose to solve their differences by a settling of accounts. Three categories of conflicts between offenders were identified. The 444 cases of settling of accounts ending with 531 victims that occured in Quebec from 1970 to 1986 were analyzed within those categories. The findings indicate that these homicides occured in cases where the stakes were very high. This study also shows why certain criminal activities and certain illicit markets give rise to more settling of accounts than others.
\end{abstract}

\section{INTRODUCTION}

La majorité des participants à des activités criminelles ont commis leurs délits avec d'autres criminels (Reiss, 1980). Les interactions qui résultent de la participation de ces individus au crime sont donc nombreuses, de même que les possibilités de conflit découlant de leur participation à un crime ou à une transaction illégale. Tous les conflits entre les délinquants ne provoquent pas l'homicide d'un des protagonistes, mais certains le font. Dans cet article, les règlements de comptes désignent cette catégorie d'homicide.

Il est manifeste que tous les conflits entre les délinquants ne se terminent pas par un règlement de comptes. En fait, il est plus juste de penser qu'une minorité de différends se règlent de cette façon. Bien que les délinquants n'aient pas accès aux tribunaux légaux, il existe des méthodes alternatives de solution des conflits. Roumasset et Hadreas (1983) par exemple, ont noté l'ostracisme dont étaient l'objet les héroï-

1. Cette recherche a été subventionnée par le Fonds pour la formation de chercheurs et l'aide à la recherche (FCAR) et par une bourse d'études du Conseil de recherches en sciences humaines (CRSH) du Canada. L'auteur tient à remercier le commandant Jacques Cloutier et le sergent Raymond Dorval de la Sûreté du Québec, ainsi que le directeur André Tessier du Service de police de la Communauté urbaine de Montréal de leur collaboration indispensable à la réalisation de ce projet. Il tient aussi à remercier Yvan Clermont, Charles Delagrave, Serge Godin et Pierre McDuff pour leur participation à la cueillette des données.

2. Agent de recherche, Centre international de criminologie comparée, Université de Montréal, case postale 6128, succursale A, Montréal, Québec, H3C 3J7. 
nomanes soupçonnés d'être des informateurs de police: en refusant d'acheter leur marchandise, les receleurs contraignaient les hérö̈nomanes à vendre ailleurs et à rabais. D'autre part, Reuter (1984) a compilé un certain nombre d'indices tendant à dérnontrer l'existence de «tribunaux d'arbitrage» administrés par la mafia NewYorkaise. Ces «tribunaux» seraient, selon Reuter, mis à la disposition des individus impliqués dans les affaires de la mafia.

En dépit de l'existence d'alternatives, le recours à l'intimidation et à la violence pour résoudre les conflits se pose comme la solution la plus évidente: tous les délinquants ne sont pas en mesure d'infliger les «sanctions économiques» mentionnées par Roumasset et Hadreas (1983), ni de mobiliser les éventuelles ressources d'arbitrage de la mafia. Par contre, les règlements de comptes ne sont que la manifestation la plus extrême de cette forme de violence qui peut être administrée à des doses plus réduites.

Dans cet article, nous examinerons pourquoi et dans quelles circonstances les délinquants choisissent les règlements de comptes pour résoudre les différends qui les opposent. Comme le soulignent Cornish et Clarke (1987: 935), la décision de commettre un crime spécifique découle d'un processus par lequel le délinquant évalue les mérites relatifs d'un ensemble d'actions qui lui permettraient d'atteindre ses objectifs. Il se livre ainsi à une analyse (souvent superficielle et rudimentaire) des coûts et des bénéfices propres à chacun des modes d'action possibles, et choisit, compte tenu de ses propres aptitudes, celui qu'il estime être le plus rentable.

Suivant cette approche, on peut relever immédiatement au moins deux facteurs pouvant mener à la décision de commettre un règlement de comptes. D'abord, le délinquant qui choisit ce mode d'action doit sans doute posséder une certaine expérience de la violence (il doit être en mesure de tuer de sang froid); ensuite, compte tenu de l'importance des risques associés à cette activité (notamment celui d'être arrêté et incarcéré pour 25 ans), les bénéfices escomptés du règlement de comptes doivent être élevés. Sans perdre de vue la première dimension, nous explorerons plus à fond la seconde, celle des gains, qui se traduit ici par l'importance des enjeux des conflits opposant les délinquants: plus ceux-ci sont élevés, plus le règlement de comptes apparaît comme une solution rentable et valable.

Dans une première section de cet article, nous identifions et regroupons en trois catégories l'ensemble des conflits qui opposent les délinquants entre eux. Ensuite, dans la mesure où la nature des enjeux 
varie selon les types de conflits identifiés, nous examinons pour chacun d'eux les enjeux des conflits qui provoquent des règlements de comptes. Cette analyse permet de comprendre dans quelles situations les règlements de comptes sont utilisés pour résoudre les conflits et indique pourquoi certaines activités criminelles et certains marchés illicites provoquent plus de règlements de comptes que d'autres.

\section{LES DONNÉES}

Notre banque de données touche l'ensemble des règlements de comptes ayant eu lieu au Québec entre 1970 et 1986. Au Québec, la seule banque de données centralisée (autre que les statistiques) sur l'homicide est le journal Allô Police. Bien qu'une grande partie de cet hebdomadaire spécialisé dans l'homicide offre un contenu résolument sensationnel, les informations factuelles sont fiables, comme l'ont d'ailleurs démontré les archives policières que nous avons consultées par la suite.

Fondé en 1953, Allô Police est devenu au fil des ans le plus important répertoire des homicides commis dans la province de Québec et ses dossiers sont régulièrement consultés par les policiers. Charland (1976) a examiné jusqu'à quel point la «revue annuelle des meurtres» (qui donne un sommaire des homicides commis durant l'année) offerte par Allô Police était complète. En la comparant aux statistiques officielles sur l'homicide, Charland a trouvé que seulement $10 \%$ de ceux-ci nétaient pas inclus dans la «revue». Toutefois, cet écart était dû surtout au fait que tous les homicides couverts par Allô Police pendant l'année n'étaient pas inclus dans les revues annuelles des meurtres. Pour neutraliser cette inadéquation, nous avons consulté tous les numéros du journal de 1970 à 1986.

Cette consultation avait pour but de repérer, parmi l'ensemble des homicides, tous les règlements de comptes ayant eu lieu durant cette période. Pour ce faire, nous avons d'abord éliminé tous les meurtres qui ne pouvaient pas être des règlements de comptes (les homicides familiaux, passionnels, les pactes de suicide, les homicides commis au cours d'un vol, les infanticides, etc.). Cette première épuration nous a conduit à retenir un ensemble d'événements incluant une variété de meurtres non résolus et d'homicides dont on allègue qu'ils sont liés à la pègre, ainsi que des cas d'incendies criminels et de bagarres de taverne ayant mal tourné. 
Dans la mesure où la plupart des règlements de comptes ne sont jamais résolus par la police, nous avons ensuite développé une série de critères pour la sélection finale des règlements de comptes au journal Allô Police. Ces critères sont les suivants : la disposition du corps de la victime; les circonstances précédant le meurtre; le modus operandi (armes utilisées; lieu du meurtre, etc.); le statut de la victime (était-elle connue de la police ?); et enfin, l'opinion des enquêteurs telle que rapportée par Allo Police. Un bon candidat pour notre définition de règlement de comptes était, par exemple, le cas du corps criblé de balles (modus operandi) d'un trafiquant de drogues connu des policiers (statut de la victime), trouvé enroulé dans un tapis placé dans le coffre arrière de sa voiture (disposition du corps), considéré par les enquêteurs interviewés comme la victime d'un règlement de comptes. Bien entendu, tous les cas ne sont pas aussi clairs. Par contre, l'exemple ci-dessus est plutôt routinier et permet de classer l'incident comme un règlement de comptes même si l'affaire n'est pas résolue et que l'événement spécifique à l'origine de l'homicide n'est pas connu.

Ce recensement mené à partir des archives du journal Allô Police a permis de retenir un premier ensemble de 464 événements considérés comme des règlements de comptes plausibles. Dans une deuxième étape, les archives policières furent mises à profit. L'accès aux dossiers d'homicides de la Sûreté du Québec (district de Montréal) et du Service de police de la communauté urbaine de Montréal nous a permis de consulter les rapports d'enquêtes de $339(73 \%)$ des 464 affaires retenues. Le reste des dossiers n'ont pu être consultés soit parce qu'ils étaient dispersés dans l'ensemble de la province, soit parce qu'ils contenaient, au moment de la collecte des données, des informations nouvelles jugées particulièrement «délicates» par les corps policiers chargés de l'enquête. Une comparaison entre les rapports consultés et les informations tirées du journal Allô Police a conduit à l'élimination de $4,3 \%$ des affaires : quelques cas avaient été considérés à tort comme des règlements de comptes, alors que d'autres affichaient un manque d'informations, ou des informations trop ambiguës, ce qui rendait possibles les interprétations alternatives. Suite à cette demière épuration, un ensemble de 444 événements ont été retenus.

Enfin, pour déterminer avec quel degré de certitude les affaires non-résolues pouvaient être, sur la base de nos critères, correctement classées comme des règlements de comptes, les dossiers d'enquête eux-mêmes furent soumis à un test de fiabilité. Quelques-uns des cas retenus (en fait 76 ou $17 \%$ ) n'étaient pas résolus au temps (T1) de l'homicide, mais l'ont été plusieurs années plus tard (au temps T2), sur- 
tout à cause des informations additionnelles fournies par des tueurs devenus délateurs. Dans ces cas, la classification opérée avec les données disponibles au temps Tl a été confirmée par les données additionnelles du temps $\mathrm{T} 2$. Ceci suggère que les critères utilisés pour identifier les règlements de comptes parmi l'ensemble des homicides (surtout les cas non-résolus) étaient raisonnablement efficaces.

Notre banque de données est donc consituée de 444 affaires de règlements de comptes ayant fait 531 victimes. Parmi l'ensemble de celles-ci, $11 \%$ (58) n'étaient pas les cibles visées lors de l'homicide (la plupart étaient des témoins gênants). Ce qui nous laisse donc avec un ensemble de 444 affaires ayant fait 473 victimes-cibles. Pour chacune des affaires, nous avons recueilli des informations sur les victimes et les tueurs (âge, sexe, antécédents judiciaires, appartenance à des groupes criminels organisés), sur l'homicide proprement dit (date, heure, endroit, modus operandi, etc.), sur le mobile du meurtre et sur les suites judiciaires de l'événement.

\section{LES CONFLITS ENTRE DÉLINQUANTS}

L'objectif de cette section est d'examiner quels sont les conflits qui opposent les délinquants entre eux. Furstenberg (1969) et Reuter (1983) ont examiné le rôle joué par la violence dans les activités des groupes criminels organisés. Ils ont élaboré des typologies des conflits entre les participants au crime. Mais leurs classifications des conflits s'appuient en grande partie sur la qualité hiérarchique des protagonistes. Ainsi, un conflit de même nature est considéré différemment selon qu'il implique un «patron» et un «employé» ou deux employés d'une même organisation criminelle. Ces deux classifications s'adressent spécifiquement aux différends impliquant des groupes criminels hiérarchisés, en fait, le crime organisé. Or, les marchés criminels et ces organisations ne détiennent le monopole ni du crime, ni des conflits, ni de la violence.

Les conflits entre délinquants peuvent se produire à l'intérieur comme à l'extérieur des groupes organisés. Une typologie générale des conflits doit donc s'appuyer sur des caractéristiques communes à l'ensemble des activités délictueuses, indépendamment de l'appartenance des protagonistes à des groupes criminels organisés, ou de la hiérarchie à l'intérieur de ces groupes. Ce ne sont pas les groupes qui provoquent les conflits, ce sont les comportements. Dans ces conditions, il est préférable de regrouper les conflits selon les genres de 
comportements qui les provoquent, indépendamment de l'implication des participants à des groupes criminels organisés.

Comme le soulignent Moore (1977) et Reuter (1984) la participation à des marchés illicites se distingue de la participation à des marchés légaux d'au moins deux façons. D'abord, par le fait que les participants aux activités criminelles risquent l'arrestation et la condamnation. Ensuite, par l'absence de contrats écrits, légalement exécutoires. Ces deux caractéristiques ne sont pas spécifiques aux marchés illicites, ni au crime organisé, mais s'appliquent à l'ensemble des activités criminelles: tous les participants au crime risquent d'être arrêtés ou d'être victimes, sans recours légal, de partenaires qui ne respectent pas leurs engagements. Ces deux types de risques servent de point de départ à une typologie des conflits susceptibles de se produire entre les participants au crime.

On peut d'abord regrouper les comportements qui exposent les délinquants à l'intervention de la justice. Ces comportements qui augmentent les risques d'arrestation et de condamnation constituent une première source de conflits spécifiques aux activités délinquantes. Bien que la délation ne soit pas le seul comportement susceptible d'augmenter ces risques, l'expression «conflits de délation» désignera cette catégorie.

L'absence de contrats écrits est une seconde source de conflits entre délinquants. Ceux-ci sont impliqués dans toutes sortes de transactions : en vue de commettre un crime (division du travail avant un vol à main armée), transactions en vue de profiter du crime (avec un receleur par exemple), transactions comportant des biens ou des services interdits (trafic d'armes, de drogue, prostitution), etc. Toutes ces transactions sont des sources potentielles de mésententes et les délinquants ne disposent pas de moyens légaux de faire respecter leurs ententes. Si un délinquant est fraudé ou volé par un partenaire, un employé, un patron, un fournisseur ou un consommateur, il devra se faire justice lui-même. Ces comportements, qui tiennent de la délinquance des délinquants entre eux, sont à l'origine de ce que nous nommerons les conflits transactionnels.

Les délinquants ne font pas que se protéger de la police et des autres délinquants : il leur faut aussi «faire du crime» d'une manière qui soit profitable. Or les profits retirés par les délinquants de certains crimes, notamment les trafics de toutes sortes, dépendent de l'étendue de leur clientèle. On peut considérer que les individus impliqués dans ces délits font du commerce et qu'ils sont exposés à la compétition des 
autres délinquants. Cette situation provoque les conflits compétitifs, qui se produisent quand un délinquant, ou un groupe, tente de s'emparer du «commerce» illégal d'un autre délinquant ou d'un autre groupe.

Ces trois types de conflits sont susceptibles de produire des règlements de comptes. Le tableau I montre l'incidence des affaires de règlements de comptes (indépendamment du nombre de victimes par affaire) pour chacun des types identifiés. On remarque que les conflits ne sont pas connus dans environ la moitié des cas. Mais l'application des critères de repérage énumérés à la section précédente laisse peu de doutes quant à leur statut de règlements de comptes.

\section{TABLEAU I}

Distribution des règlements de comptes selon les types de conflits et l'issue judiciaire

\begin{tabular}{lcr}
\hline CONFLITS & N & $\%$ \\
\hline DÉLATION & 52 & $24,4 \%$ \\
TRANSACTIONNELS & 84 & $39,4 \%$ \\
COMPÉTITION & 77 & $36,2 \%$ \\
\hline TOTAL (connus) & 213 & $100,0 \%$ \\
\hline INCONNUS & 231 & $52,0 \%$ \\
\hline TOTAL & 444 & $100,0 \%$ \\
\hline
\end{tabular}

Le tableau I montre que les conflits de délation, transactionnels et de compétition sont responsables respectivement de $24,4 \%, 39,4 \%$ et $36,2 \%$ des affaires de règlements de comptes dont l'origine est connue. Toutefois, ce tableau n'indique pas l'incidence respective des conflits entre les délinquants. Il se pourrait, par exemple, que $50 \%$ des différends soient causés par la délation et $10 \%$ par des conflits transactionnels, mais que ces derniers se terminent plus fréquemment par des règlements de comptes.

\section{LES CONFLITS QUI PROVOQUENT DES RÈGLEMENTS DE COMPTES}

Dans cette section, nous examinerons dans quelles situations les règlements de comptes sont utilisés par les délinquants et, par la même occasion, dans quelles situations ils ne le sont pas. Par définition, tous 
les crimes dont la commission implique la participation de plusieurs délinquants risquent de provoquer des conflits qui se résolvent par des règlements de comptes. Cependant, le règlement de comptes est en soi une activité dangereuse: environ $25 \%$ de ces homicides donnent lieu, tôt ou tard, à des accusations. Compte tenu du fait qu'un individu choisissant de résoudre un conflit de cette manière court un risque non négligeable d'être accusé de meurtre, on s'attend à ce que les règlements de comptes soient utilisés surtout quand les enjeux des conflits sont très importants.

L'évaluation de l'importance des enjeux varie selon la nature des conflits. Le préjudice causé par la délation dépend de la sévérité de la sanction pénale que celui qui a été dénoncé risque de se voir infliger. Les enjeux d'un conflit transactionnel sont quant à eux associés à l'importance monétaire de la transaction litigieuse. Enfin, l'importance des enjeux d'un conflit compétitif dépend de la valeur du marché criminel, ou de la partie de marché qui en fait l'objet. L'identification des secteurs d'activités criminelles qui provoquent des conflits meurtriers constitue un point de départ à l'évaluation de l'importance des enjeux : certains délits et certains marchés criminels sont plus rentables que d'autres et certains crimes sont plus sévèrement punis que d'autres. Nous examinerons donc, pour chacun des trois types de conflits identifiés, quelles sont les activités criminelles qui provoquent les règlements de comptes.

\section{LA DÉLATION}

Un soir de novembre 1977, Serge Labrosse ${ }^{3}$ sortait d'un cabaret de Montréal en état d'ébriété. Le lendemain matin, on le trouvait mort, étranglé avec sa cravate, sur la banquette avant de sa voiture garée dans le stationnement du club de nuit. Labrosse, qui avait participé au vol d'un camion remorque contenant $430000 \$$ de cigarettes, s'était fait arrêter à Sherbrooke, alors qu'il transmettait la marchandise. Suite à son arrestation, il avait choisi de dénoncer ses complices. Dans cette affaire, Labrosse s'est fait tuer parce qu'il avait trahi ses complices afin de faire réduire les accusations portées contre lui.

La trahison de Serge Labrosse a incité ses complices à se venger en l'éliminant. Comme nous l'avons souligné cependant, la délation proprement dite n'est pas le seul comportement qui augmente les risques d'arrestation et d'incarcération: les agissernents de complices trop voyants peuvent être aussi compromettants et provoquer des

3. Les événements relatés sont réels. Seuls les dates et les noms ont été changés. 
conflits qui se terminent en règlements de comptes. Nos données indiquent toutefois que seulement trois $(5,8 \%)$ des 52 règlements de comptes de notre catégorie délation peuvent être imputés à ce genre de comportement. Puisque la quasi-totalité des événements de cette catégorie sont des cas de délation, l'analyse portera sur ces derniers. Après avoir formulé quelques remarques générales sur les situations dans lesquelles ils sont commis, l'examen des crimes à l'origine des règlements de comptes liés à la délation permettra d'évaluer l'importance des enjeux.

Nos données permettent d'identifier deux caractéristiques des règlements de comptes liés à la délation: d'abord, les victimes sont des délateurs, plutôt que des indicateurs; ensuite, les règlements de comptes peuvent avoir une fonction préventive aussi bien que de vengeance.

1. On peut regrouper les délinquants qui coopèrent avec la police en deux groupes: les indicateurs qui donnent des informations au sujet de délits auxquels ils n'ont pas participé ; et les délateurs qui dénoncent leurs complices. Les deux groupes ont des rôles différents: les indicateurs mettent les enquêteurs sur la piste des délinquants, tandis que les délateurs, par leur témoignage, contribuent à alourdir les preuves contre leurs ex-complices. Les données suggèrent que ce sont les délateurs, plutôt que les indicateurs, qui sont victimes de règlements de comptes: dans toutes les affaires connues de délation, les accusés étaient les complices des victimes. Il se pourrait par contre que des victimes d'affaires pour lesquelles on ignore quel conflit est à l'origine du règlement de comptes, aient été des indicateurs. Mais cette éventualité apparaît peu probable pour les raisons suivantes: d'une part, le caractère anonyme de leur comportement garantit une immunité relative aux indicateurs. Le fait qu'ils n'aient pas participé au crime dont ils dénoncent l'auteur assure que leur identité restera secrète : la partie lésée peut difficilement faire le rapprochement entre un indicateur quelconque et la trahison dont il a été l'objet; d'autre part, il est plus facile pour la police de cacher l'identité d'un indicateur que celle d'un délateur. D'abord les délateurs sont souvent appelés à témoigner au procès de leurs complices. Ensuite, ils peuvent bénéficier d'un traitement privilégié, une sentence réduite par exemple, en échange de leur collaboration.

2. En matière de délation, un délinquant peut entrer en conflit avec un autre soit parce qu'il a été dénoncé, soit parce qu'il le soupçonne de pouvoir et de vouloir le faire. Dans le deuxième cas, on peut dire du règlement de comptes qu'il a des visées préventives: les victimes sont tuées avant qu'elles ne parlent. Notons du reste que les trois règle- 
ments de comptes causés par le comportement trop voyant des victimes peuvent être considérés comme des homicides préventifs. En ajoutant ces trois cas aux 49 cas de délation proprement dits, on observe que la moitié $(48,1 \%)$ des règlements de comptes de cette catégorie ont été faits par mesure préventive.

\section{LES CRIMES DÉNONCÉS}

Les enjeux d'un conflit de délation sont associés à la sévérité de la peine reçue ou escomptée suite à la dénonciation faite par un complice bavard : plus la sentence est sévère, plus les enjeux sont importants. On s'attend donc à ce que les règlements de comptes soient le plus probables quand les crimes dénoncés sont sévèrement punis.

TABLEAU II

Distribution des règlements de comptes liés à la délation selon les délits à l'origine des conflits

\begin{tabular}{lrr}
\hline DÉLITS* & N & $\%$ \\
\hline DROGUE** & 10 & $22,7 \%$ \\
VOL À MAIN ARMÉE & 13 & $29,5 \%$ \\
HOMICIDE & 14 & $31,8 \%$ \\
VOL & 2 & $4,5 \%$ \\
AUTRES & 5 & $11,4 \%$ \\
\hline SOUS-TOTAL & 44 & $100 \%$ \\
\hline NCONNU & 8 & $15,4 \%$ \\
\hline TOTAL & 52 & $100 \%$ \\
\hline
\end{tabular}

* Chaque catégorie de délit inclut les tentatives

** Toutes les infractions relatives aux stupéfiants

Le tableau II montre que les homicides et les vols à main armée sont à l'origine de $61 \%$ des règlements de comptes liés à la délation. Le fait que ces deux délits sont plus sévèrement punis que les autres semble confirmer notre hypothèse. Les homicides, notamment, comptent pour seulement $0,03 \%$ des crimes connus (Statistique Canada, 1982a), mais sont responsables de $31,8 \%$ des règlements de comptes liés à la délation. Si on la compare au vol sans violence, la probabilité qu'un participant à un homicide soit éventuellement tué par ses complices est environ 12000 fois plus élevée 4 .

4. Il se commet, selon les données de Statistique Canada (1982a), 1700 fois plus de vols sans violence que d'homicides ( $52 \%$ des crimes rapportés contre $0,03 \%$ ), mais ceuxci provoquent sept fois plus de règlements de comptes (cf. tableau II). C'est donc dire que le meurtre d'un participant à un homicide a environ 12000 fois $(1700 \times 7)$ plus de chances de se produire que celui d'un voleur. 
On remarque aussi que les délits de drogue sont responsables de $22,7 \%$ des règlements de comptes liés à la délation. À première vue, cette statistique pourrait étonner. En effet, comme le souligne Skolnick (1967: 137),

narcotics crimes are typically without citizen complainants and therefore require the development of an information system for the apprehension of narcotics offenders.

On devrait donc s'attendre à ce que le trafic de stupéfiants produise une plus grande quantité d'informateurs que celui du vol à main armée et de l'homicide. De plus, le préjudice causé par la délation ne se limite pas ici aux sentences reçues ou éventuelles. Même si celles-ci sont moins sévères que pour les homicides et le vol à main armée, il faut aussi compter que les pertes financières associées à une saisie de drogue peuvent être importantes. Ainsi, la délation en matière de stupéfiants semble être un phénomène relativement courant et les préjudices causés peuvent être importants. Il peut donc sembler étonnant que ces délits ne provoquent pas plus de règlements de comptes.

La nature du délit, et surtout des interventions policières et des preuves nécessaires à la condamnation des trafiquants, peuvent expliquer la proportion relativement faible de règlements de comptes liés à la drogue dans cette catégorie. Il est très difficile de condamner un trafiquant si on ne l'arrête pas pendant la transaction, ou pendant qu'il est en possession de la drogue. Les informateurs sont utilisés pour renseigner les enquêteurs sur les lieux de la transaction ou de l'entreposage. Une fois les renseignements obtenus, les policiers procèdent aux arrestations, saisissent la drogue et n'ont plus besoin des informateurs, les trafiquants étant arrêtés en flagrant délit. Les informateurs n'ont donc pas à témoigner et leur identité restera secrète. Il en est de même pour les cas de «buy and bust» où un policier achète des stupéfiants d'un fournisseur avant de l'arrêter. Le trafiquant sait qu'il a été vendu, mais il ne peut pas savoir par qui. Ceci pourrait expliquer la sous-représentation relative des délits en matière de stupéfiants parmi les règlements de comptes liés à la délation.

\section{LES CONFLITS TRANSACTIONNELS}

À Montréal, un soir de veille de Noël, Réjean et Paul Couturier sont tués de plusieurs balles de revolver de calibre ,22 et ,38 alors qu'ils sont dans la voiture de Réjean. Les victimes sont connues de la police pour leur participation à des vols à main armée, ainsi que leur 
implication dans le prêt usuraire et le racket de la protection. Suite au meurtre, les enquêteurs concluent que les victimes ont doublé trois complices lors du partage $\mathrm{d} \mu$ butin d'un vol à main armée. Bien que l'on soupçonne ces derniers d'être les auteurs du double règlement de comptes, la police n'a pas assez de preuves pour les incriminer. $\mathrm{Ce}$ n'est que 12 ans plus tard que l'un des meurtriers, devenu délateur, sera condamné pour ce crime. Les deux autres étant décédés entre-temps, aucune accusation n'a été portée contre eux.

Nos données indiquent que $39,4 \%$ des règlements de comptes dont on connaît le mobile sont provoqués par des conflits transactionnels (cf. tableau I). Le meurtre des frères Couturier donne un exemple des mésententes qui peuvent survenir dans les transactions criminelles. Les conflits de cet ordre peuvent se produire dans toutes les activités criminelles comportant des transactions et des ententes verbales ou tacites (puisqu'il n'y a pas de contrats écrits ni d'instance formelle d'arbitrage). Par exemple, le client qui ne respecte pas ses engagements auprès d'un usurier, l'acheteur de drogue qui refuse de payer son fournisseur, le fournisseur qui fraude un client sur la qualité ou la quantité de la drogue vendue, le collecteur d'un usurier ou d'un preneur au livre qui lui vole de l'argent et l'employeur qui refuse de payer un tueur à gages pour le travail accompli sont tous des sources de mésententes susceptibles de provoquer des règlements de comptes.

Avant de considérer le facteur de l'importance des enjeux, quatre caractéristiques des règlements de comptes liés aux conflits transactionnels seront soulignées.

1. Nos données sur le sujet ne sont pas assez détaillées pour permettre d'évaluer exactement l'ampleur du phénomène, mais elles indiquent néanmoins que les règlements de comptes sont souvent précédés de menaces adressées aux victimes par la partie lésée dans un conflit transactionnel. Ces menaces visent à récupérer les biens ou l'argent subtilisés par la victime. Dans ces cas, le meurtre peut être considéré comme une mesure de dernier recours utilisée quand les tentatives de récupération ont échoué. Les menaces préliminaires sont en fait assez compréhensibles: en tuant la victime, le meurtrier abandonne l'espoir d'être dédommagé pour le tort qu'il a subi.

2. Cette caractéristique des règlements de comptes provoqués par des conflits transactionnels soulève la question suivante : quel est l'intérêt d'éliminer quelqu'un si on perd tout espoir de récupérer son dû? 
Sans perdre de vue la vengeance, signalons l'existence d'une explication plus mercantile. En effet, comme le souligne Dietz (1983: 89),

criminal business is handled without recourse to courts and written contracts so in order to conduct business there must be a combination of trust and the threat of force. In a situation where someone takes money from another, or humiliates that person publicly, the person loses face. If they do not take forceful action, they will be perceived as fair game to be ripped off by anyone. Thus, in order to maintain the business, action must be taken.

Selon cette interprétation, les règlements de comptes générés par les conflits transactionnels auraient une fonction dissuasive. Un élément essentiel des transactions criminelles est la menace de représailles si une des parties ne respecte pas ses engagements. Or, pour que la menace soit crédible, elle doit, au moins parfois, être mise à exécution. Ainsi, l'usurier qui tue un client mauvais payeur perd l'argent qui lui était dû. Par contre, si le mauvais payeur n'est pas tué, ses autres clients peuvent penser qu'ils peuvent ne pas payer en toute sécurité. L'usurier perdra donc plus s'il ne tue pas le mauvais payeur.

3. Une conséquence de la formulation de menaces préliminaires au règlement de comptes est qu'il arrive que la partie lésée d'un conflit transactionnel soit elle-même tuée. En prenant les devants, le délinquant menacé élimine à la fois ses dettes et les menaces de mort qui lui sont adressées.

4. Soulignons enfin que certains règlements de comptes échappent à cette dynamique de la menace. Il peut arriver, par exemple, qu'un délinquant en tue un autre sans avertissement pour effacer ses dettes, mais les règlements de comptes sans menaces préliminaires semblent surtout faire des victimes chez les vendeurs de drogue qui se font voler: pendant la transaction, l'acheteur tue le vendeur et s'enfuit avec la drogue (mentionnons qu'il arrive que ce soit l'acheteur qui se fasse tuer par le vendeur qui veut lui voler l'argent de la transaction).

\section{LES ENJEUX DES CONFLITS TRANSACTIONNELS}

En matière de conflits transactionnels, l'importance des enjeux est associée aux pertes financières occasionnées par les différends euxmêmes, auxquelles peuvent s'ajouter, comme le souligne Dietz (1983), les pertes futures découlant des dommages à la réputation. 
TABLEAU III

Distribution des règlements de comptes liés aux conflits transactionnels selon les délits à l'origine des conflits

\begin{tabular}{lrr}
\hline DÉLITS* & $\mathrm{N}$ & $\%$ \\
\hline DROGUE** & 48 & $61,5 \%$ \\
VOL À MAIN ARMÉE & 21 & $26,9 \%$ \\
HOMICIDE & 1 & $1,3 \%$ \\
VOL & 2 & $2,6 \%$ \\
AUTRES & 6 & $7,7 \%$ \\
\hline SOUS-TOTAL & 78 & $100,0 \%$ \\
\hline INCONNU & 6 & $7,1 \%$ \\
\hline TOTAL & 84 & $100,0 \%$ \\
\hline
\end{tabular}

* Chaque catégorie de délit inclut les tentatives

** Toutes les infractions relatives aux stupéfiants

Le tableau III montre la distribution des règlements de comptes liés aux conflits transactionnels selon les délits à l'origine des conflits. On remarque que $26,9 \%$ des règlements de comptes découlent de conflits liés au mauvais partage du butin de vols à main armée, comparativement à 2,6\% pour les vols simples. En supposant que les risques d'occurrence d'un conflit suite à un vol à main armée et suite à un vol simple soient les mêmes, alors les mésententes sur le partage du butin seraient beaucoup plus meurtrières pour les vols à main armée. Ainsi, ceux-ci représentent $0,65 \%$ des crimes connus, tandis que les vols comptent pour $52 \%$ (Statistique Canada, 1982a). Il se commet donc 80 fois plus de vols sans violence que de vols à main armée, mais ces derniers font environ 10 fois plus de victimes de règlements de comptes. Notons que ces données sous-estiment probablement la surreprésentation du vol à main armée. dans la mesure où, comme le souligne West (1979), les délinquants qui commettent les crimes les plus graves et les plus violents auront tendance à être plus «réguliers» entre eux, compte tenu de leur capacité de recourir à la violence. Ainsi, un conflit serait plus susceptible d'éclater suite à un vol sans violence que suite à un vol à main armée, où les participants savent que leurs complices peuvent recourir à la violence.

Le fait que les participants aux vols à main armée aient une certaine expérience de la violence, qu'ils possèdent des armes et qu'ils savent s'en servir pourrait être un élément important dans le choix du règlement de comptes comme mode de solution d'un conflit. Il est 
cependant plus difficile d'évaluer l'importance des enjeux des conflits puisque nous ne connaissons que rarement ( 6 cas sur 21) les sommes impliquées dans les vols à main armée qui en sont à l'origine. Mais dans les six cas où ces montants sont connus, ceux-ci variaient entre $50000 \$$ et $175000 \$$, pour une moyenne de $120833 \$$. À titre comparatif, les données de Marceau (1982: 38) indiquent que seulement $2,1 \%$ d'un échantillon aléatoire des vols à main armée commis avec une arme à feu à Montréal en 1979, ont procuré des gains supérieurs à $10000 \$$ aux délinquants, tandis que la médiane des montants volés se situe aux environs de $100 \$$. Bien que nos données en la matière puissent être biaisées (si par exemple les montants des vols n'étaient mentionnés dans les dossiers d'enquête que lorsqu'ils sont élevés), les différences observées entre les vols à main armée «typiques», tels que décrits par Marceau (1982), et ceux qui provoquent les règlements de comptes sont si marquées qu'elles supportent l'idée que l'importance des enjeux est un facteur non négligeable dans l'issue des conflits.

Le tableau III montre également que la majorité $(61,5 \%)$ des règlements de comptes provoqués par des conflits transactionnels sont liés au trafic de drogue. Il est plus difficile d'évaluer l'importance monétaire relative des enjeux de ces conflits puisque nous ne disposons pas de données permettant d'évaluer la valeur d'une transaction de drogue moyenne. Notons toutefois que les 18 conflits liés à la drogue pour lesquels nous connaissons l'importance des enjeux indiquent que la valeur des montants impliqués varie entre $500 \$$ et $305000 \$$, pour une moyenne de $57500 \$$ et une médiane de $15000 \$$ par conflit. Ces données doivent être interprétées avec prudence, mais elles suggèrent que les règlements de comptes ne sont généralement pas l'affaire des petits trafiquants de rue.

Les enjeux des transactions litigieuses ne sont probablement pas le seul élément qui entre en ligne de compte dans le domaine de la drogue. Il se pourrait bien que le nombre élevé de transactions explique pourquoi la majorité des conflits transactionnels qui se terminent par un règlement de comptes sont liés à la drogue. Les sondages de délinquance autorévélée menés auprès de détenus américains montrent que le nombre médian de vols qualifiés qu'ils commettent annuellement varie de trois (pour les détenus du Texas) à huit (pour les détenus californiens), tandis que le nombre médian de transactions de drogue varie de 36 pour les détenus texans à 166 pour les Californiens (Blumstein $e t$ al., 1986: 314-315; Chaiken et Chaiken, 1982). De plus, les infractions relatives aux drogues représentent $3 \%$ de toutes les infractions au code criminel et aux statuts fédéraux rapportées à la police, comparativement 
à $0,65 \%$ pour les vols qualifiés (Statistique Canada, 1982a). En tenant compte du chiffre noir, que l'on soupçonne considérable en matière de trafic de drogue, on peut conclure que les transactions de drogue sont beaucoup plus fréquentes que les vols qualifiés. Par conséquent, les risques qu'une transaction de drogue provoque un conflit qui se termine par un règlement de comptes seraient moins élevés que pour le vol à main armée. Et la raison pour laquelle la majorité des règlements de comptes sont liés au trafic de drogue serait le nombre élevé de transactions.

\section{LES CONFLITS ASSOCIÉS À LA COMPÉTITION}

Pierre Gendron et Guy Pinard sont assis dans un bar de Montréal au moment où entre Georges Neveu. Gendron invite alors Pinard et Neveu chez lui dans le nord de la ville. Les trois prennent place sur la banquette avant de la voiture conduite par Pinard. Au moment où la voiture passe dans un coin isolé de Montréal-Nord, Gendron poignarde Neveu au coeur à plusieurs reprises. Avant de s'enfuir, Pinard arrête la voiture et Gendron pousse le cadavre de Neveu dans la rue. En dépit des avertissements répétés des hommes de main du groupe auquel sont affiliés Gendron et Pinard, Georges Neveu vendait toujours de la drogue dans un établissement où le groupe écoulait sa propre marchandise.

Comme l'illustre cet exemple, les règlements de comptes sont parfois utilisés pour mettre un terme à un conflit généré par la compétition que les délinquants se livrent pour l'exploitation d'un territoire. Les conflits de nature compétitive sont responsables de $36 \%$ des règlements de comptes (cf. tableau I) et, la plupart du temps, ceux-ci impliquent des groupes. Les conflits peuvent opposer un groupe à un individu (comme dans l'exemple qui précède) ou deux groupes entre eux, mais rarement deux individus isolés.

Parce que ces différends impliquent des groupes, les conflits liés à la compétition sont susceptibles de faire plusieurs victimes. Pour les conflits de délation et les conflits transactionnels, la plupart du temps, le règlement de comptes marque la fin du conflit. Par contre, puisque les conflits de compétition impliquent des groupes, le règlement de comptes ne marque pas nécessairement la fin du conflit. Il y a des risques de représailles, comme l'indique le fait que 54 des 77 affaires identifiées comme des conflits de compétition dérivent en fait de 6 conflits opposant des groupes. Chacun de ces conflits ayant fait en moyenne 9 règlements de comptes. Il y a donc peu de conflits meurtriers liés à la compétition, mais ceux-ci font beaucoup de victimes: les 
guerres de gangs sont provoquées par des conflits de nature compétitive et chaque guerre découle, en fait, d'un seul conflit.

\section{LES ENJEUX DES MARCHÉS}

Par définition, les conflits liés à la compétition ont pour objet le contrôle de l'ensemble ou d'une partie d'un marché criminel. Les enjeux de ces conflits peuvent donc d'emblée être considérếs importants. Le tableau IV montre que $83 \%$ des règlements de comptes compétitifs sont associés au marché de la drogue 5 . On peut penser que tous les marchés de drogue ne sont pas également rentables (la cocaiine vs la marijuana par exemple). De même, les profits varient sans doute selon les niveaux de distribution à l'intérieur des marchés : les enjeux ne sont pas les mêmes pour l'importateur de cocaïne et pour le trafiquant de rue. Nos données ne permettent pas de faire ces distinctions, mais on peut penser que les marchés de drogues, pris dans leur ensemble, sont plus profitables que les autres marchés criminels.

\section{TABLEAU IV}

Distribution des règlements de comptes liés aux conflits de compétition selon les délits à l'origine des conflits

\begin{tabular}{lrr}
\hline DÉLITS & $\mathrm{N}$ & $\%$ \\
\hline DROGUE & 56 & $83,6 \%$ \\
VOL À MAIN ARMÉE & 0 & $0,0 \%$ \\
HOMICIDE & 0 & $0,0 \%$ \\
VOL & 0 & $0,0 \%$ \\
AUTRES* & 11 & $16,4 \%$ \\
\hline SOUS-TOTAL & 67 & $100,0 \%$ \\
\hline INCONNU & 10 & $13,0 \%$ \\
\hline TOTAL & 77 & $100,0 \%$ \\
\hline
\end{tabular}

* Les 11 règlements de comptes inclus dans la catégorie «autres» sont associés au marché du prêt usuraire. En fait, il s'agit d'une guerre de gang (la guerre de l'Ouest de Montréal, en 1974-75) qui fut déclenchée entre deux groupes qui voulaient s'approprier le commerce d'un usurier.

5. Les 10 règlements de comptes classés dans la catégorie «délits inconnus» avaient pour objet le contrôle de territoires où les délinquants peuvent s'adonner à plusieurs activités. Par exemple, le contrôle d'un bar permet d'y vendre sa drogue, d'opérer un commerce de prêt usuraire, de contrôler la prostitution, d'y écouler des biens volés, etc. Les 83\% (56) de règlements de comptes compétitifs liés à la drogue sont donc une estimation minimale de la place que ce marché occupe parmi l'ensemble des règlements de comptes compétitifs. 
Ceci ne veut pas dire que les autres marchés criminels ne sont pas suffisamment rentables pour susciter la convoitise de compétiteurs. Ainsi, comme le montre Leguerrier (1989a), le propriétaire d'une entreprise de prostitution commerciale (un salon de massage) peut espérer un gain moyen de 48000 \$ net avant de fermer ses portes, généralement au bout d'une année d'opération (l'espérance de vie moyenne de ces entreprises). Or, comme nous l'avons vu dans la section précédente, cette somme s'apparente aux enjeux moyens des règlements de comptes liés à la drogue provoqués par les conflits transactionnels (57500 \$). Par ailleurs, les travaux de Leguerrier (1989b) suggèrent que le marché des pièces d'automobiles volées assure à ses entrepreneurs, des revenus considérablement plus élevés que ceux de la prostitution commerciale. Il semble donc que la rentabilité de ces marchés (et probablement de plusieurs autres) pourrait justifier, théoriquement du moins, l'usage des règlements de comptes compétitifs.

Les gains escomptés (les enjeux) par celui qui déclenche les hostilités dans un conflit compétitif ne dépendent pas tant du chiffre d'affaires de l'entrepreneur agressé, que de la nouvelle clientèle qu'il espère recruter. Pour les marchés de drogue, le règlement de comptes permet, s'il produit un effet décisif, de s'emparer directement du commerce et de la clientèle du compétiteur éliminé. Or, ce n'est le cas ni de la prostitution commerciale, ni du recel de voitures volées. Leguerrier (1989a, 1989b) souligne que ces marchés criminels ne peuvent opérer sans le recours à des entreprises de façade légales (les studios de massage pour la prostitution commerciale), ou sans être intégrés aux opérations d'entreprises légitimes (les commerces de pièces d'automobiles usagées ou recyclées pour le recel de voitures volées). Or, l'élimination d'un concurrent dans ces marchés criminels ne donne pas l'accès à l'entreprise légale qu'il opère. C'est donc dire que le règlement de comptes ne permettrait pas de s'emparer directement du commerce et de la clientèle d'un compétiteur.

Dans les marchés criminels opérant sous le couvert d'entreprises licites, les seuls gains espérés du meurtre d'un compétiteur proviendraient d'un déplacement de la clientèle. Le décès d'un propriétaire de salon de massage provoquerait la fermeture de son établissement, qui se traduirait par une augmentation du chiffre d'affaires de l'agresseur. L'usage du règlement de comptes à cette fin est hautement improbable pour deux raisons. D'abord, l'entrepreneur dispose de méthodes alternatives beaucoup moins risquées pour accroître sa clientèle: il peut par exemple adopter une stratégie publicitaire plus aggressive, ou encore, plus simplement, forcer la fermeture d'une entreprise rivale en dénon- 
çant son compétiteur à la police. Ensuite, en supposant, généreusement, que l'élimination d'un compétiteur provoque un accroissement de clientèle de $25 \%$ chez le meurtrier, et que l'entreprise de ce dernier soit en opération depuis six mois, il peut espérer, selon la durée de vie moyenne de ce type d'entreprise, des gains supplémentaires de $6000 \$ 6$. Dans ces conditions, le règlement de comptes n'apparaît pas comme une solution attrayante.

Dans le domaine du recel des pièces de voitures volées, le meurtre d'un entrepreneur rival ne provoque pas la fermeture de son commerce. Le recel de pièces d'autos volées se fait dans le cadre des opérations légales d'entreprises de recyclage. Ces entreprises ne sont pas des façades, dans la mesure où la majorité de leurs opérations sont légales. Advenant le décès du propriétaire, l'entreprise peut être prise en charge par sa succession ou être vendue, mais elle poursuit ses opérations. La seule manière d'en assurer le contrôle est de s'en porter acquéreur. On comprend alors que les règlements de comptes ne rapporteraient, à toute fin pratique, rien au meurtrier.

Chaque marché criminel présente des caractéristiques qui lui sont propres. Aussi, les conclusions que l'on peut tirer de notre analyse peuvent ne pas s'appliquer à l'ensemble des marchés. Mais, l'étude comparée des marchés de la prostitution et $\mathrm{du}$ recel de pièces de voitures volées et du marché de la drogue, suggère que les marchés criminels opérant au moyen de façades d'affaires légales, ou dans le cadre d'entreprises légitimes, ne sont pas un terrain propice à la violence compétitive, ou à tout le moins, aux règlements de comptes. Dans ces marchés criminels, les enjeux ne semblent pas justifier l'usage de l'assassinat: une entreprise opérant derrière une façade légale peut être très profitable pour son propriétaire, mais le meurtre de celui-ci ne permet pas de s'emparer de son commerce et de sa clientèle. On comprend alors que les règlements de comptes de nature compétitive ne se produisent que dans les marchés criminels qui ne nécéssitent pas le concours d'entreprises légalement constituées. Ces marchés sont peu nombreux : les stupéfiants; la prostitution de rue et le prêt usuraire.

6. Cette estimation s'appuie sur les données de Leguerrier (1989a): ces entreprises ont une espérance de vie moyenne d'un an: rapportent $4000 \$$ par mois à leurs propriétaires; et le taux annuel de fermeture était, en 1985 (la demière année d'observation de Leguerrier) de $50 \%$ (ces fermetures sont causées soit par des faillites et des départs volontaires $(26 \%)$, soit par des opérations policières $(24 \%)$. 


\section{CONCLUSION}

Dans cet article, nous avons vu que les conflits entre délinquants sont les plus susceptibles de se terminer par des règlements de comptes lorsque les enjeux sont importants et lorsque l'homicide offre, au moins en apparence, une certaine rentabilité pour le meurtrier. Ainsi, les règlements de comptes sont plus probables lorsqu'une délation implique un meurtre antérieur pour lequel les participants risquent l'emprisonnement à vie (ou y sont déjà condamnés), que lorsqu'elle implique un cambriolage; les participants à un vol à main armée impliquant des sommes importantes sont plus susceptibles de s'entretuer que ceux qui n'ont récolté qu'un maigre butin; et le règlement de comptes compétitif est utilisé dans les marchés de drogue plutôt que dans ceux de la prostitution commerciale et du recel de voitures volées en raison des gains plus importants que peut en retirer l'agresseur.

L'importance des enjeux semble donc peser de manière décisive sur l'issue d'un conflit. Il est par contre manifeste que ce n'est pas le seul facteur en cause. Il se pourrait d'abord que les règlements de comptes ne soient utilisés que quand aucune méthode autre que la violence n'est accessible. Rappelons aussi que selon l'hypothèse de Cornish et Clarke (1987), formulée au début de cet article, les délinquants choisissent les modes d'action les plus rentables compte tenu de leurs propres aptitudes. Nous avons observé que les règlements de comptes liés à la délation étaient provoqués en majorité par des conflits faisant suite à des homicides et à des vols à main armée (cf. tableau II). L'hypothèse de l'importance des enjeux permet, comme nous l'avons vu, d'expliquer ce phénomène. Par contre, il ne faut pas oublier que les participants aux homicides et aux vols qualifiés ont une certaine expérience de la violence, qu'ils possèdent des armes et qu'ils savent s'en servir. Cette expérience pourrait exercer un impact tout aussi important que les enjeux sur le choix du règlement de comptes comme mode de solution d'un conflit. De même, si l'importance des enjeux peut expliquer que les règlements de comptes provoqués par des conflits transactionnels liés à des vols qualifiés se produisent quand le butin du vol est élevé, ce facteur n'explique pas l'absence de règlements de comptes liés à la fraude, ni la sous représentation des vols sans violence (deux délits qui peuvent être aussi rentables que le vol qualifié et présenter des enjeux aussi importants) dans cette catégorie (cf. tableau III). Il est donc très plausible que le facteur de l'importance des enjeux et les aptitudes des protagonistes, qui se traduisent ici par une expérience des solutions violentes et la possession d'armes meurtrières, agissent de concert dans la décision de commettre un règlement de comptes. Dans 
les cas où les enjeux d'un conflit sont importants, cette solution serait alors plus accessible à ceux qui possèdent cette expérience.

Enfin, l'examen des circonstances qui entourent la commission des règlements de comptes conduit à une réflexion plus large sur l'ensemble du milieu criminel. Il est évident que les conflits dont l'issue est fatale ne représentent qu'une minorité des différends qui opposent les délinquants entre eux. Il se pourrait bien que l'image du milieu criminel léguée par Sutherland (1937) ne reflète plus la réalité contemporaine. À eux seuls, l'incidence des règlements de comptes et les risques auxquels s'exposent les délinquants ${ }^{7}$ remettent en cause l'image d'un milieu criminel où les relations entre participants logent à l'enseigne de la franche camaderie, où les bandes concurrentes rivalisent de courtoisie et où «les exemples de délation sont si rares qu'il est à peine nécessaire d'en faire mention» (Sutherland, 1937: 16). L'analyse des règlements de comptes remet aussi en question l'image d'un milieu criminel «organisé» que décrivait Cressey (1969). L'incidence des règlements de comptes compétitifs liés aux marchés de la drogue n'est possible, comme l'a souligné Reuter (1983) que dans un marché concurrentiel, désorganisé et peuplé de petites entreprises relativement éphémères. Quant à l'absence de règlement de comptes dans les autres marchés criminels, elle ne constitue pas un témoignage de leur haut niveau d'organisation, mais reflète plutôt l'insignifiance des gains qu'un agresseur peut espérer retirer d'un conflit meurtrier.

\section{RÉFÉRENCES}

BLUMSTEIN, A., COHEN, J., ROTH, J. A., VISHER, C. A. (1986), Criminal Careers and "Career Criminals". Washington D.C. : National Academy Press.

CHAIKEN, J. M., CHAIKEN, M. R. (1982), Varieties of Criminal Behavior, Santa Monica, Calif. : Rand Corporation.

CHARLAND, R. (1976), Le meurtre à Montréal de 1944 à 1975 : une étude descriptive, mémoire de maîtrise es sciences (criminologie), École de Criminologie : Université de Montréal, 301 pages.

CORDEAU, G. (1989), Les règlements de comptes au Québec et les mécanismes de la dissuasion endogène, Revue canadienne de Criminologie, vol. 31, $\mathrm{n}^{\circ} 3,253-279$.

7. La probabilité qu'un participant au crime soit victime d'un règlement de comptes en une année donnée est estimée à 0,0011 (Cordeau, 1989), soit 22 fois plus élevée que celle qu'un mâle adulte soit victime d'un meurtre au Québec en 1981 (140 victimes mâles adultes (Statistique Canada, 1982b), dont 30 par règlements de comptes pour une population de 2218764 (Statistique Canada, 1982c) donnent une probabilité de 0,00005 (110/population). 
CORNISH, D.B., CLARKE, R.V. (1987), Understanding Crime Displacement : An Application of Rational Choice Theory, Criminology, vol. 23, $\mathrm{n}^{\circ} 4,933-947$.

CRESSEY, D. (1969), Theft of the Nation: The Structure and Operation of Organized Crime in America, New York: Harper and Row.

DIETZ, M. L. (1983), Killing for Profit, Chicago: Nelson-Hall.

FURSTENBERG, M. (1969), Violence in Organized Crime p. 911-939 in Crimes of Violence, Staff Report to the Commission on the Causes and Prevention of Violence, vol. 13, Washington D.C. : U.S. Government Printing Office.

LEGUERRIER, Y. (1989a), Les entreprises de prostitution commerciale : les commerces éphémères des marchés illicites, Criminologie, vol. XXII, no 2.

LEGUERRIER, Y. (1989b), Le vol de véhicules automobiles : le cas de deux marchés contrastés, article soumis à la Revue canadienne de Criminologie.

MARCEAU, B. (1982), Le vol à main armée à Montréal, p. 4-83 in Normandeau, A., Le vol à main armée au Québec : études empiriques préliminaires, rapport technique $n^{\circ} 2$, Centre international de criminologie comparée : Université de Montréal.

MOORE, M. (1977), Buy and Bust, Lexington, Mass. : D.C. Heath

REISS, A. J. (1980), Understanding Changes in Crime Rates, p. 11-17 in Indicators of Crime and Criminal Justice : Quantitative Studies, edited by Stefen E. Fienberg and Albert J. Reiss, U.S. Department of Justice.

REUTER, P. (1983), Disorganized Crime: The Economics of the Visible Hand, Cambridge, Mass. : The MIT Press.

REUTER, P. (1984), Social Control in Illegal Markets, p. 29-58 in Toward a General Theory of Social Control, Donald Black (ed.), New York : Academic Press.

ROUMASSET, J., HADREAS, J. (1977), Addicts, Fences and the Market for Stolen Goods, Public Finance Quarterly, vol. 5, $\mathrm{n}^{\circ} 2,247-272$.

SKOLNICK, J. H. (1967), Justice Without Trial: Law Enforcement in Democratic Society, New York : Wiley.

STATISTIQUE CANADA (1982a), Crime and Trafic Enforcement Statistics, catalogue 85-205, Ottawa: Minister of Supply and Services Canada.

STATISTIQUE CANADA (1982b), Statistique de l'homicide (I981), catalogue 85-209. ministre des Approvisionnements et services, Ottawa.

STATISTIQUE CANADA (1982c), Divisions et subdivisions de recensement. Populations, logements privés occupés, ménages privés, familles de recensement dans les ménages privés. Certaines caractéristiques, Québec 1981, Ottawa.

SUTHERLAND, E. H. (1937), Le voleur professionnel, Paris : Spes (traduction française, 1963).

WEST, W. G. (1979), Trust Among Serious Thieves, Crime etland Justice, vol 8, $\mathbf{n}^{\circ} 4$, 239-246. 